\title{
Modelling the Connectivity of Natura 2000 Sites and Their Integration in the Wider Landscape
}

\author{
Christine ESTREGUIL and Giovanni CAUDULLO \\ Joint Research Centre of the European Commission, Ispra/Italy · christine.estreguil@jrc.ec.europa.eu
}

This contribution was double-blind reviewed as extended abstract.

\section{Extended Abstract}

Within the European Biodiversity Strategy for 2020 (EC 2011), the Green Infrastructure (GI) is introduced as one supporting tool to increase spatial and functional connectivity between natural protected and un-protected areas, and to improve landscape permeability (DG ENV. 2012). Natura 2000 (N2K) sites network, designated under the "Habitat" Directive (EC, 1992) and the "Bird" Directive (EC 2010), will form the backbone of GI. Connectivity is defined as a combined product of structural and functional connectivity, i.e. the effect of physical landscape structure and the actual species use of the landscape.

The proposed integrated model is based on two available free software packages (GUIDOS at forest.jrc.ec.europa.eu/download/software/guidos from SOILLE and VOGT 2009 and Conefor at www.conefor.org from SAURA and TORNÉ 2009) and new Python programming tools for automation of model procedures operating spatial data with ESRI Arcpy libraries. It is applied to assess and report the connectivity of N2K sites network in a harmonized, easily reproducible and automated manner on the basis of both structural and functional criteria. The approach represents a compromise between a pure functional biological model and the commonly used connectedness measure for assessment over large regions (e.g. OPERMANIS et al. 2012).

Data input to the model was the European N2K spatial and descriptive dataset (DG ENV. 2012) from which only N2K sites including forest habitats were considered. In order to address functional (least cost) distance between sites, a European-wide land use based friction map was created as a proxy of landscape resistance by using a logarithmic friction scale from 1 to 1,000 per distance unit $(1 \mathrm{~m})$ depending on land uses. It was based on the Corine Land Cover map of the year 2006, which was reclassified into three land cover classes (artificial, intensive agriculture, natural) and was further enriched by the fine resolution Open Street Map (www.openstreetmap.org): highways, motorways, national roads were considered barriers for terrestrial wildlife movement; bridges, viaducts and tunnels, which act as points facilitating species movements, were accounted for.

The structural connectivity of the N2K network was described in terms of simple subnet (one node or site) and complex subnets (interconnected nodes or site with links) (Fig. 1) from the Morphological Spatial Pattern Application (MSPA) of GUIDOS software. The functional connectivity of the $\mathrm{N} 2 \mathrm{~K}$ network was then addressed by using the Conefor software on the basis of the size of each site, their topology and inter-site effective (least cost) distances for a given species dispersal ability. Two indices - the site area weighted 
Root Probability of Connectivity (RPC), and the un-weighted Average Probability of Connectivity (APC) which is more sensitive to the inter-site landscape resistance and functional distances between subnets - were derived from the simplified power weighted probability of dispersal function introduced in ESTREGUIL et al. (2012). The probability of dispersal decreases as an exponential function of the effective distance and the landscape resistance, computed from the land use based friction map with the "least cost path" method. The cost distance matching the $50 \%$ probability of dispersal was 50,000 , which corresponds to the average dispersal distance $(500 \mathrm{~m})$ of most 'connectivity sensitive' forest dwelling species, multiplied by the average friction per distance unit (100). Functional subnets were defined as those from which at least one least cost path was found to another subnet and for those the average of active probabilities of dispersal was given (active P mean). The N2K share in functionally isolated subnets, i.e. those from which no least cost paths were found, was also provided.

Table 1: Results of the structural connectivity analysis for 11 selected countries in Europe: country based share of Natura 2000 sites in simple and complex subnets

\begin{tabular}{|l|c|c|c|c|c|c|c|c|c|c|c|}
\hline Country & AT & DE & ES & HU & IE & IT & LV & NL & PO & SK & SE \\
\hline $\begin{array}{l}\text { Simple subnet } \\
\text { (N2K \%) }\end{array}$ & 80.4 & 82.9 & 77.8 & 80.4 & 80.6 & 87.1 & 94.9 & 80.4 & 84.7 & 90.4 & 93.8 \\
\hline $\begin{array}{l}\text { Complex subnet } \\
\text { (N2K \%) }\end{array}$ & 19.6 & 17.1 & 22.2 & 19.6 & 19.4 & 12.9 & 5.1 & 19.6 & 15.3 & 9.6 & 6.2 \\
\hline
\end{tabular}

Results on the intra-connectivity (Fig. 1 and Tab. 1) and inter-site connectivity (Fig. 2 and 3) of all N2K sites including forest habitats are presented for eleven countries in Europe, allowing for comparison across countries. The average of active probabilities of dispersal ("Active P mean" on row 2 of table underneath Fig. 3) accounts only for all functionally connected subnets and translates the landscape resistance along the least cost path linking each pair of subnets. Furthermore, the analysis quantifies the N2K sites share in functionally isolated sites ("Isolated subnet" on row 3 of the table underneath the chart in Figure 3) and also enables their geo-location (map not shown). Once identified, the physical and/or functional isolation of protected $\mathrm{N} 2 \mathrm{~K}$ sites may be mitigated by decreasing landscape resistance and distances in between protected sites and/or creating new protected sites where needed. The analysis could provide guidance for a regional landscape planning of clearings and re/afforestation measures to enhance the whole connectivity of the network. 


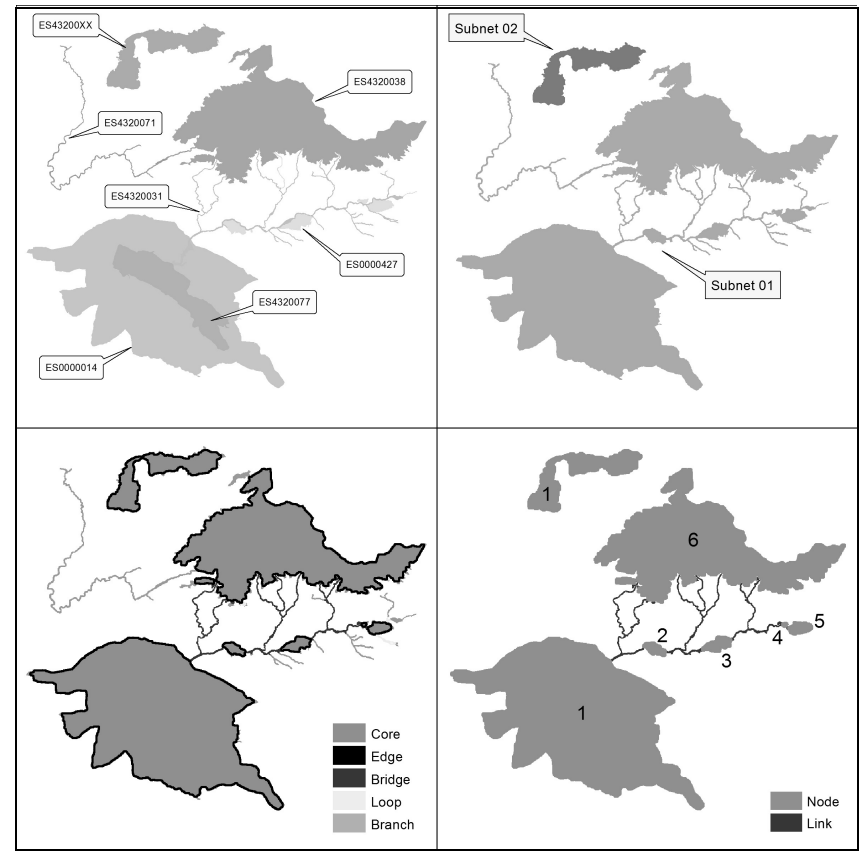

Fig. 1: Structural connectivity analysis of a complex and a simple sub-network: (topleft) subset of seven N2K sites; (top-right) binary masking; (bottom-left) morphological map after GUIDOS MSPA processing and (bottom-right) subnets categorisation into 2 subnets: simple subnet (1 node) and complex subnet (6 nodes and 4 links).

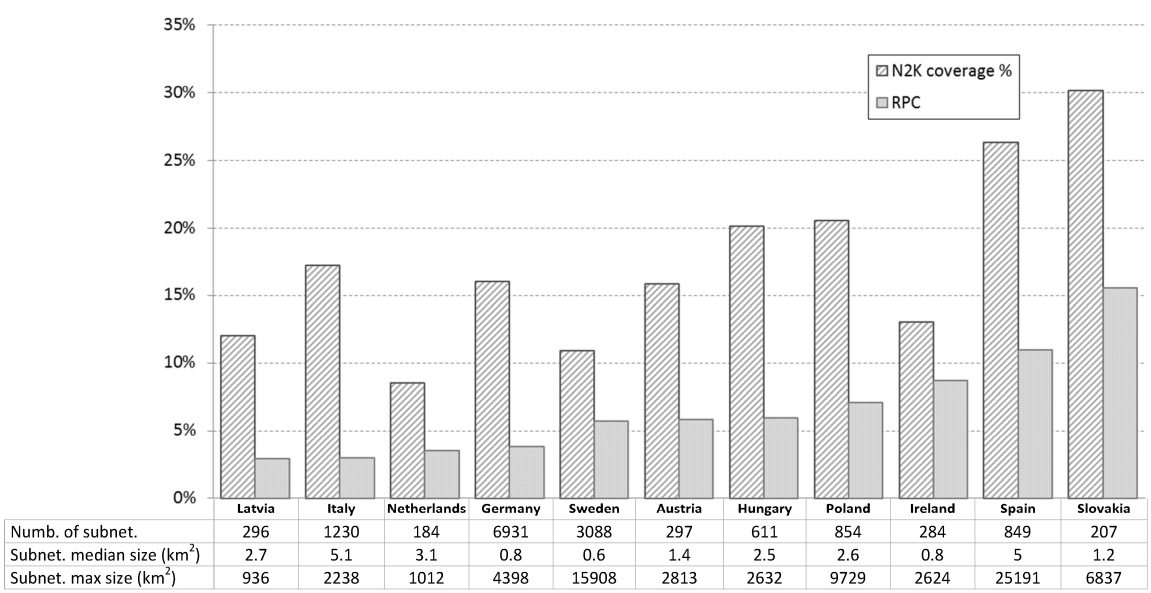

Fig. 2: Site area weighted functional connectivity (RPC) of all N2K sites incl. forest for 11 countries: the RPC range is between $0 \%$ and the maximum connectivity equivalent to the N2K land proportion. The number, median size and maximum size of subnets are indicated per country for subsequent analysis. 


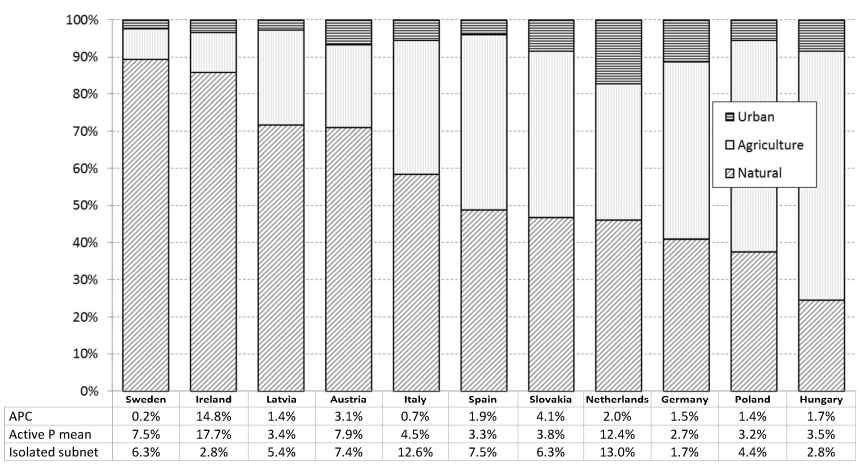

Fig. 3: Inter-site landscape composition for 11 countries ranked by decreasing natural/semi-natural land proportion; the table provides the un-weighted functional connectivity (APC) index, the average of active probabilities of dispersal (Active P mean) and the proportion of isolated subnets.

This paper is an attempt to respond partly to the methodological needs for European-wide broad scale assessment of connectivity of $\mathrm{N} 2 \mathrm{~K}$ sites in the GI context, where increasing spatial and functional connectivity between protected and un-protected areas is one objective.

\section{References}

DG ENV (2012), Science for Environment Policy. In-depth report on 'The Multifunctionality of Green Infrastructure.

http://ec.europa.eu/environment/nature/ecosystems/docs/Green_Infrastructure.pdf

EC (2011), Our life insurance, our natural capital: an EU biodiversity strategy to 2020. COM (2011) 244 final.

http://ec.europa.eu/environment/nature/biodiversity/comm2006/pdf/2020/1_EN_ACT_p art1_v7\%5B1\%5D.pdf.

Estreguil, C., Caudullo, G., De Rigo, D., Whitmore C.\& San-Miguel-Ayanz J. (2012), Reporting on European forest fragmentation: Standardised indices and web map services. IEEE Earthzine. On line Earthzine's Forest Resource Information theme in second quarter 2012. http://www.earthzine.org/?p=384031.

Opermanis, O., MacSharry B., Aunins, A., Sipkova, Z. (2012), Connectedness and connectivity of the Natura 2000 network of protected areas across country borders in the European Union. Biological Conservation 153, 227-238.

DOI:10.1016/j.biocon.2012.04.031.

SAURA, S. \& TORNÉ, J. (2009), Conefor Sensinode 2.2: a software package for quantifying the importance of habitat patches for landscape connectivity. Environmental Modelling \& Software, 24, 135-139.

Sollle, P. \& VoGT, P. (2009), Morphological segmentation of binary patterns. Patterns Recognition Letters, 30 (4), 456-459. DOI: 10.1016/j.patrec.2008.10.015. 